\title{
EXPLORING THE X-RAY AND $\gamma$-RAY PROPERTIES OF THE REDBACK MILLISECOND PULSAR PSR J1723-2837
}

\author{
C. Y. Hui ${ }^{1}$, P. H. T. TAm ${ }^{2}$, J. Takata ${ }^{3}$, A. K. H. Kong ${ }^{2}$, K. S. Cheng ${ }^{3}$, J. H. K. Wu ${ }^{2}$, L. C. C. Lin ${ }^{4}$, And E. M. H. Wu ${ }^{3}$ \\ ${ }^{1}$ Department of Astronomy and Space Science, Chungnam National University, Daejeon 305-764, Korea \\ ${ }^{2}$ Institute of Astronomy and Department of Physics, National Tsing Hua University, Hsinchu, Taiwan \\ ${ }^{3}$ Department of Physics, University of Hong Kong, Pokfulam Road, Hong Kong \\ ${ }^{4}$ General Education Center, China Medical University, Taichung 40402, Taiwan \\ Received 2013 November 1; accepted 2013 December 23; published 2014 January 6
}

\begin{abstract}
We have investigated the X-ray and $\gamma$-ray properties of the redback millisecond pulsar PSR J1723-2837 with $X M M$-Newton, Chandra, and Fermi. We have discovered the X-ray orbital modulation of this binary system with a minimum that coincides with the phases of radio eclipse. The X-ray emission is clearly non-thermal in nature, which can be described well by a simple power law with a photon index of $\sim 1.2$. The phase-averaged luminosity is $\sim 9 \times 10^{31} \mathrm{erg} \mathrm{s}^{-1}$ in $0.3-10 \mathrm{keV}$, which consumes $\sim 0.2 \%$ of the spin-down power. We have detected the $\gamma$-ray emission in $0.1-300 \mathrm{GeV}$ from this system at a significance of $\sim 6 \sigma$ for the first time. The $\gamma$-rays in this energy range consume $\sim 2 \%$ of the spin-down power and can be modeled by a power law with a photon index of $\sim 2.6$. We discuss the high energy properties of the new redback in the context of an intrabinary shock model.
\end{abstract}

Key words: gamma rays: stars - pulsars: individual (PSR J1723-2837) - X-rays: binaries

Online-only material: color figure

\section{INTRODUCTION}

In the last few years, a new population of eclipsing binary millisecond pulsars (MSPs) has emerged. Although the range of orbital period spanned by these systems is similar to that of "black widow" MSPs $\left(P_{b} \lesssim 20 \mathrm{hr}\right)$, their companion masses $\left(M_{c} \sim 0.2-0.4 M_{\odot}\right)$ are significantly larger than those of black widows $\left(M_{c} \ll 0.1 M_{\odot}\right.$; Roberts 2013). This population is dubbed as "redbacks." Chen et al. (2013) suggest that the determining factor for producing black widows or redbacks is the efficiency of companion evaporation. They further argue that redback systems do not evolve into black widows with time.

More than a dozen of redbacks have been identified so far. $^{5}$ PSR J1023+0038 is the first identified redback MSP that provides evidence for a transition from an $\mathrm{X}$-ray binary to a radio MSP (Archibald et al. 2009, 2010; Thorstensen \& Armstrong 2005). These systems are suggested to possibly have their states swinging between the rotation and accretion power phases according to the mass transfer rate (Shvartsman 1970; Burderi et al. 2001). Recent observations of episodic accretion from the redbacks PSR J1023+0038 (Patruno et al. 2013; Takata et al. 2013) and PSR J1824-2452I (Papitto et al. 2013) are consistent with this scenario.

The radio and optical properties of PSR J1723-2837, which is a redback, were reported recently (Crawford et al. 2013). The dispersion measure suggests it is located at a distance of $0.75 \pm 0.10 \mathrm{pc}$ (Crawford et al. 2013). Its rotational period and orbital period are $1.86 \mathrm{~ms}$ and $14.8 \mathrm{hr}$, respectively. The spin-down luminosity of the pulsar is estimated as $\dot{E}=$ $4.6 \times 10^{34} \mathrm{erg} \mathrm{s}^{-1}$. The mass of its companion lies in the range of $0.4-0.7 M_{\odot}$ and optical spectroscopy indicates that it is a G-type star. The pulsar follows a nearly circular orbit. The eclipse of the radio pulses takes $\sim 15 \%$ of the orbit, which is twice the Roche lobe size inferred for the companion (Crawford

5 See http://apatruno.wordpress.com/about/millisecond-pulsar-catalogue/ for up-to-date information. et al. 2013). In this Letter, we report the results from our X-ray and $\gamma$-ray analyses of this system.

\section{OBSERVATION AND DATA ANALYSIS}

\subsection{XMM-Newton Observations}

PSR J1723-2837 was observed by XMM-Newton on 2011 March 3 for a total exposure of $\sim 56 \mathrm{ks}$ with all EPIC cameras operating in the full frame mode (Obs. ID: 0653830101), which provides a fairly uniform orbital coverage. With the updated instrumental calibrations, we generated all of the event files with $X M M$ Science Analysis Software (XMM-SAS version 12.0.1). We selected only the data in the energy range of $0.3-10 \mathrm{keV}$ and those events correspond to the pattern in the range of 0-12 for the Metal Oxide Semiconductor (MOS) $1 / 2$ cameras and $0-4$ for the PN camera. Bad pixels were also excluded. The effective exposures for MOS1, MOS2, and PN after filtering are found to be $\sim 50.7 \mathrm{ks}, \sim 49.6 \mathrm{ks}$, and $\sim 49.3 \mathrm{ks}$, respectively. Within a source region with a $20^{\prime \prime}$ radius around the pulsar timing position $\left(\right.$ R.A. $=17^{\mathrm{h}} 23^{\mathrm{m}} 23^{\mathrm{s}} .1856$ decl. $=$ $-28^{\circ} 37^{\prime} 57^{\prime \prime} .17$ (J2000)), 4993 counts, 3655 counts, and 13261 counts (source+background) are extracted from MOS1, MOS2, and PN CCDs, respectively. All of the EPIC data are found to be unaffected by CCD pile-up.

We first searched for the orbital modulation in X-rays. Before any temporal analysis was performed, we applied barycentric correction to the arrival times of all of the events by using the updated planetary ephemeris JPL DE405. We then subtracted the background in the individual camera by adopting an annular region with an inner/outer radius of $25^{\prime \prime} / 45^{\prime \prime}$ centered at the pulsar position. Although soft proton flares contaminated the data in several regions of the CCDs, examining the light curve of the aforementioned small region-of-interest (ROI) reveals no flare-like events. Therefore, we retain all of the data in order to optimize the orbital coverage for the analysis. Using the radio ephemeris for PSR J1723-2837 (Table 2 in Crawford et al. 2013), we folded the background-subtracted light curves at the 


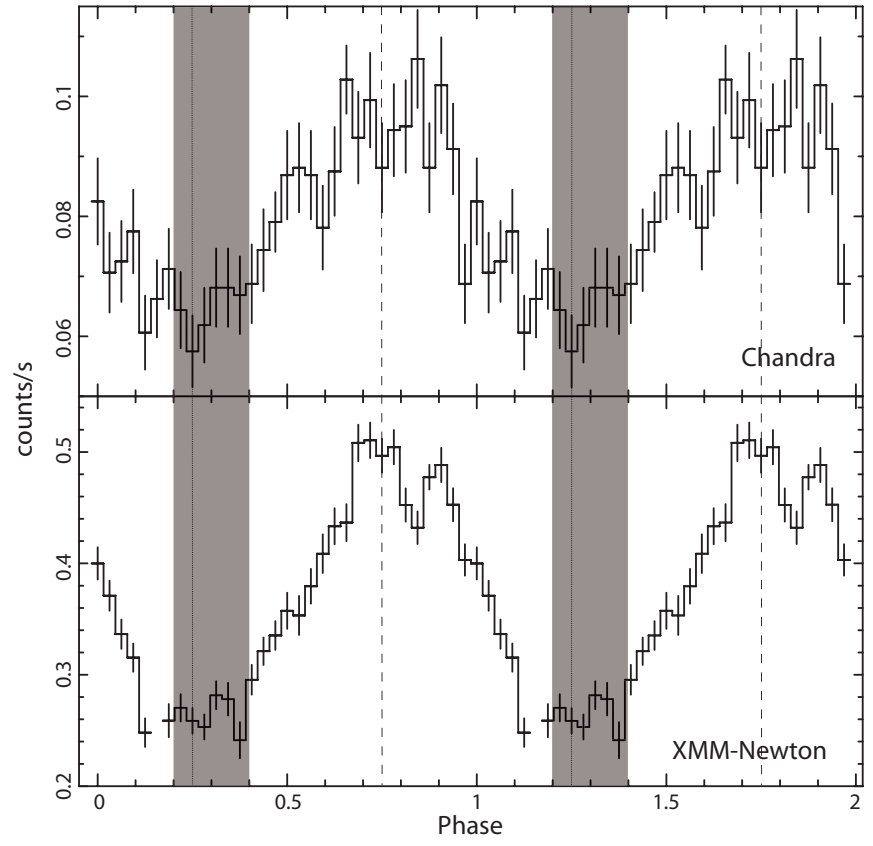

Figure 1. Background-subtracted light curves of PSR J1723-2837 as observed by Chandra ACIS in $0.3-7 \mathrm{keV}$ (upper panel) and by XMM-Newton in $0.3-10 \mathrm{keV}$ with the data from all of the EPIC cameras combined (lower panel). The same data have been repeated for another orbital cycle in order to clearly demonstrate the modulation. The shaded region illustrates the range of the radio eclipse. The dotted line and the dashed line illustrate the phases of INFC and SUPC, respectively.

orbital period. The X-ray modulation can be clearly seen with all three cameras. In order to improve the photon statistics, we combined all of the EPIC data and the resultant light curve is shown in Figure 1. We adopted the time of the ascending node (MJD 55425.320466) to be the epoch of phase zero. Due to the incomplete orbital coverage after data filtering, there is gap at the orbital phase interval $0.14-0.18$ in this XMM-Newton data.

The minimum of the X-ray orbital modulation occurs at the same phase interval at which radio eclipse has been observed in $1.4-3.1 \mathrm{GHz}$ (see Figure 1 in Crawford et al. 2013), which is illustrated by the shaded region in Figure 1. This interval encompasses the inferior conjunction (INFC) at a phase of $\sim 0.25$, where the pulsar is behind its companion. Apart from the region around the INFC, some observations at $2 \mathrm{GHz}$ performed in the phase interval of $0.5-0.6$ were also unable to detect the pulsar (Crawford et al. 2013). However, when examining the X-ray orbital modulation at this interval, we cannot identify any peculiar behavior. On the other hand, the maximum of the modulation is found in the orbital phase interval of 0.7-0.9, which encompasses the superior conjunction (SUPC; see Figure 1). In this interval, we have found a dip near a phase of $\sim 0.8$.

In order to investigate its X-ray spectral properties, we extracted the source and background spectra from the same regions adopted in the temporal analysis. We grouped the spectra obtained individually from MOS1 and MOS2 so as to have at least 50 counts per bin. For the PN spectrum, we grouped it to have at least 60 counts per bin. The spectra obtained from all three cameras are fitted simultaneously to the tested models. All of the uncertainties quoted in this paper are $1 \sigma$ for two parameters of interest (i.e., $\Delta \chi^{2}=2.3$ ).

First, we examined the phase-averaged X-ray spectrum of PSR J1723-2837. We found that a simple absorbed power-law model can describe the observed spectral data reasonably well $\left(\chi^{2}=171.84\right.$ for 179 degrees of freedom (dof)). The observed spectrum and the best-fit model are shown in Figure 2. The best-fit model yields a column density of $N_{\mathrm{H}}=2.31_{-0.25}^{+0.28} \times$ $10^{21} \mathrm{~cm}^{-2}$, a photon index of $\Gamma_{X}=1.19 \pm 0.05$, and a normalization of $1.10_{-0.07}^{+0.08} \times 10^{-4}$ photons $\mathrm{keV}^{-1} \mathrm{~cm}^{-2} \mathrm{~s}^{-1}$ at $1 \mathrm{keV}$. The best-fit $N_{\mathrm{H}}$ is consistent with the value inferred from the optical extinction $A_{V}=1.2$ of this system (Crawford et al. 2013; Predehl \& Schmitt 1995). The unabsorbed energy flux in $0.3-10 \mathrm{keV}$ is $f_{x}=1.32_{-0.16}^{+0.19} \times 10^{-12} \mathrm{erg} \mathrm{cm}^{-2} \mathrm{~s}^{-1}$. At a distance of $0.75 \mathrm{kpc}$, this corresponds to an X-ray luminosity of $L_{x} \sim 8.9 \times 10^{31} \mathrm{erg} \mathrm{s}^{-1}$. We also attempted to fit the X-ray spectrum of PSR J1723-2837 with thermal models. While a black body yields a reduced $\chi^{2}$ much larger than unity, the thermal bremsstrahlung model results in an unphysically high temperature $(k T>200 \mathrm{keV})$.

We proceeded to investigate if the spectral properties vary at different orbital phases. We have extracted the spectra from two phase ranges, 0.2-0.4 and 0.7-0.9, which encompass INFC and SUPC, respectively. For the INFC interval, the best-fit yields an $N_{\mathrm{H}}=2.94_{-1.25}^{+2.30} \times 10^{21} \mathrm{~cm}^{-2}$ and $\Gamma_{X}=1.23_{-0.19}^{+0.32}$. On the other hand, the corresponding parameters for the SUPC interval are estimated as $N_{\mathrm{H}}=2.01_{-0.43}^{+0.49} \times 10^{21} \mathrm{~cm}^{-2}$ and $\Gamma_{X}=1.09 \pm 0.09$. Within the statistical uncertainties, the spectral parameters inferred in these two intervals are consistent with the phase-averaged values. Based on this XMM-Newton observation, there is no evidence of X-ray spectral variability across the orbit of PSR J1723-2837.

\subsection{Chandra Observations}

Chandra observed PSR J1723-2837 with the Advanced CCD Imaging Spectrometer (ACIS) on 2012 July 11 (ObsID: 13713). The orbital coverage is continuous with a sum for a good time interval of $55 \mathrm{ks}$, which is slightly more than one orbital cycle. The ACIS was operated with a sub-array mode to achieve a timing resolution of $0.4 \mathrm{~s}$. Due to the loss of efficiency for the sub-array model, the effective exposure is shorter $(49.9 \mathrm{ks})$. Similar to the XMM-Newton observations, we performed barycentric correction to the arrival times of all of the events by using the updated planetary ephemeris JPL DE405. PSR J1723-2837 is clearly seen and is the brightest source in the image. In order to reduce the background, we limited our analysis to the $0.3-7 \mathrm{keV}$ band. Using a circular source region with a radius of $4^{\prime \prime}$, there are 4468 counts for subsequent analysis. An annular-source-free region centered at PSR J1723-2837 was used for background subtraction.

We extracted the 0.3-7 keV light curve of PSR J1723-2837; similarly to the XMM-Newton observation, the $14.8 \mathrm{hr}$ orbital modulation is clearly seen (see Figure 1). Although the number of counts is much lower than that of XMM-Newton, the lightcurve profile is consistent with that of XMM-Newton. In spite of the large error bars, it is worth noting that there is a hint of the dip at phase $\sim 0.8$ seen in the XMM-Newton data.

For spectral analysis, we first investigated the phase-averaged spectrum. The spectrum can be well described $\left(\chi^{2}=186.85\right.$ for 204 dof) with an absorbed power-law model with $N_{\mathrm{H}}=$ $(1.68 \pm 0.34) \times 10^{21} \mathrm{~cm}^{-2}$ and $\Gamma_{X}=1.00 \pm 0.07$. The unabsorbed $0.3-10 \mathrm{keV}$ flux is $1.64_{-0.06}^{+0.04} \times 10^{-12} \mathrm{erg} \mathrm{cm}^{-2} \mathrm{~s}^{-1}$. The spectrum of Chandra is only slightly harder than that of XMM-Newton. Since PSR J1723-2837 is bright for Chandra, some pile-up may contaminate the spectral analysis. We also included a pileup model and found that the pile-up fraction is only $1.7 \%$ and the spectral parameters are more or less the same. We then 


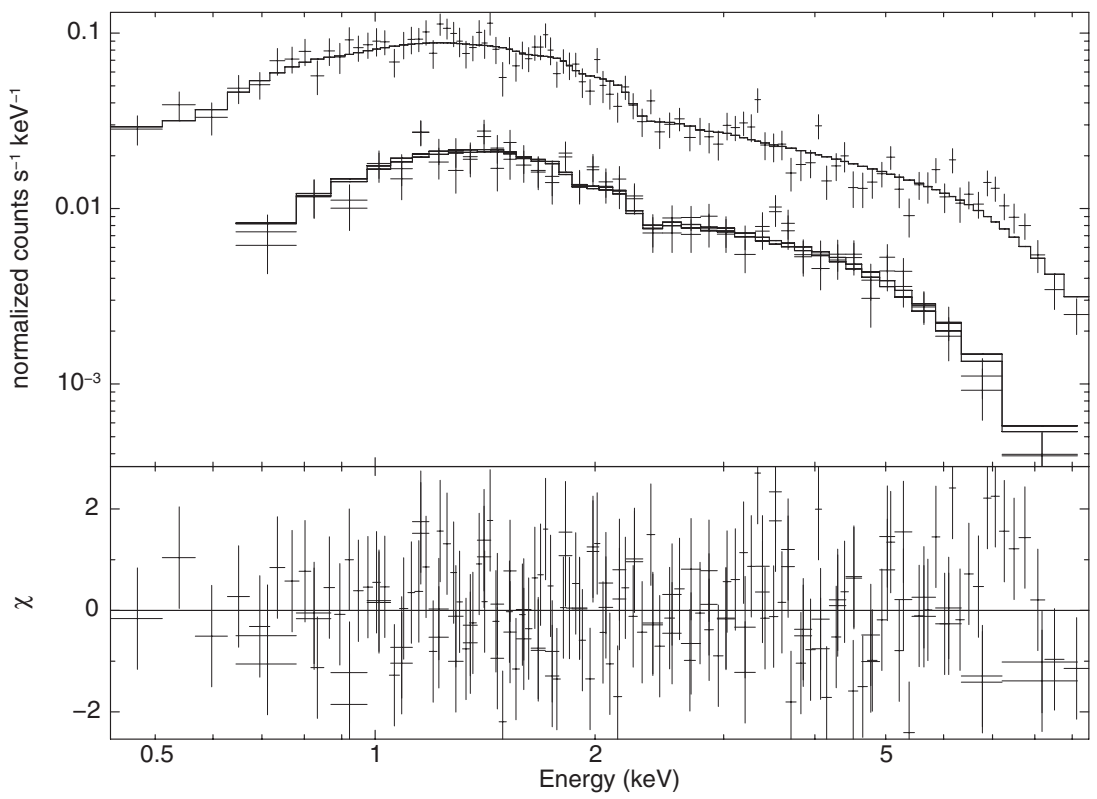

Figure 2. Phase-averaged X-ray spectra of PSR J1723-2837 as observed by XMM-Newton and simultaneously fitted to an absorbed power-law model (upper panel) and contribution to the $\chi^{2}$-fit statistic (lower panel).

performed phase-resolved spectroscopy using the same phase bins for INFC and SUPC as for the XMM-Newton data. Both spectra can be fit with an absorbed power law. For INFC, the best fit is $N_{\mathrm{H}}=\left(3.51_{-1.15}^{+1.42}\right) \times 10^{21} \mathrm{~cm}^{-2}$ and $\Gamma_{X}=1.35 \pm 0.22$, while for SUPC the corresponding parameters are $N_{\mathrm{H}}=$ $(1.3 \pm 1.0) \times 10^{21} \mathrm{~cm}^{-2}$ and $\Gamma_{X}=0.82 \pm 0.17$. Like in the $X M M-N e w t o n$ data, there is no evidence for orbital dependent spectral variability.

In order to better constrain the INFC and SUPC spectral shape, we simultaneously fitted the spectra obtained by $X M M-N e w t o n$ and Chandra in these two intervals. The crosscalibration was accounted for by allowing the normalizations to be different for these two telescopes. Assuming $N_{\mathrm{H}}$ is variable at different orbital phases, we obtained $N_{\mathrm{H}}=4.39_{-1.00}^{+1.23} \times$ $10^{21} \mathrm{~cm}^{-2}, \Gamma_{X}=1.48_{-0.14}^{+0.17}$ and $N_{\mathrm{H}}=2.01_{-0.39}^{+0.47} \times 10^{21} \mathrm{~cm}^{-2}$, $\Gamma_{X}=1.05 \pm 0.08$ for INFC and SUPC, respectively. The spectral parameters inferred in these phase intervals can be reconciled within $2 \sigma$ uncertainties. We have also explored the possibility that there is no significant variation of $N_{\mathrm{H}}$ across the orbit. We jointly fitted individual power-law models to the INFC and SUPC spectra with $N_{\mathrm{H}}$ in the individual models tied together. This yields $N_{\mathrm{H}}=2.47_{-0.42}^{+0.46} \times 10^{21} \mathrm{~cm}^{-2}$ and $\Gamma_{X}=1.23_{-0.08}^{+0.11}$, $\Gamma_{X}=1.12_{-0.04}^{+0.08}$ for INFC and SUPC, respectively. The photon indices in these two intervals are consistent within $1 \sigma$ uncertainties. Therefore, we do not find any conclusive evidence of spectral variation across the orbit.

\subsection{Fermi Large Area Telescope (LAT) Observations}

The $\gamma$-ray data used in this work were obtained between 2008 August 4 and 2013 July 15, and are available at the Fermi Science Support Center. ${ }^{6}$ We used the Fermi Science Tools v9r31p1 package to reduce and analyze the Pass7 data in the vicinity of PSR J1723-2837. To reduce the contamination from Earth albedo $\gamma$-rays, we excluded events with zenith angles greater than $100^{\circ}$. We used events with energies between $100 \mathrm{MeV}$ and $300 \mathrm{GeV}$ in the binned likelihood analysis. The corresponding instrumental response functions were used.

\footnotetext{
6 http://fermi.gsfc.nasa.gov/ssc/
}

We first chose a rectangular region of dimension $28^{\circ} \times 28^{\circ}$ centered on the radio timing position as our ROI. We subtracted the background contribution by including the galactic diffuse model (gal_2yearp7v6_v0.fits) and the isotropic background (iso_p7v6source.txt), as well as all of the sources in the second Fermi/LAT catalog (2FGL; Nolan et al. 2012) within the circular region of $25^{\circ}$ radius around PSR J1723-2837 in the background source model. We assumed the respective spectrum in the 2FGL catalog for the sources considered. The normalization of the diffuse components and spectral parameter values of sources within $5^{\circ}$ from PSR J1723-2837 were allowed to vary.

Using the "source"-class events and assuming a power-law spectrum at the pulsar position, the maximized test-statistic (TS) value (Mattox et al. 1996) we obtained for the PSR J1723-2837 position is 38.3 , corresponding to a detection significance of $6.2 \sigma$. The power-law index is $\Gamma_{\gamma}=2.6 \pm 0.1$. The photon flux from the power-law fit is $(3.5 \pm 0.7) \times 10^{-8}$ photons $\mathrm{cm}^{-2} \mathrm{~s}^{-1}$, and the energy flux is $(1.5 \pm 0.3) \times 10^{-11} \mathrm{erg} \mathrm{cm}^{-2} \mathrm{~s}^{-1}$, corresponding to a $\gamma$-ray luminosity of $L_{\gamma} \sim 10^{33} \mathrm{erg} \mathrm{s}^{-1}$ at a distance of $0.75 \mathrm{kpc}$. Since it is uncertain how much the pulsar proper motion contributes to the spin-down rate, the resulting spin-down luminosity can be treated as an upper limit of the intrinsic spin-down luminosity. Therefore, $L_{\gamma}$ would be no smaller than $\sim 2 \%$ of the intrinsic spin-down luminosity. Attempts to fit the data using a power law with an exponential cutoff at the PSR J1723-2837 position did not provide a constraining parameter set. We also chose different ROIs to verify our results.

Given the proximity of PSR J1723-2837 to the Galactic Center where the galactic $\gamma$-ray diffuse background is high, we also performed a binned likelihood analysis using "clean"-class events in a smaller ROI of dimension $21^{\circ} \times 21^{\circ}$ centered on the radio timing position. The TS value thus obtained is 24.2 , corresponding to a detection significance of $4.9 \sigma$. Taking into account the above likelihood results using "source" events and "clean" events as well as the $\gamma$-ray count map shown in Figure 3, we believe that the detection of the $\gamma$-ray source is robust.

To better estimate the $\gamma$-ray position, we performed an unbinned analysis of the front-converted "clean"-class events 


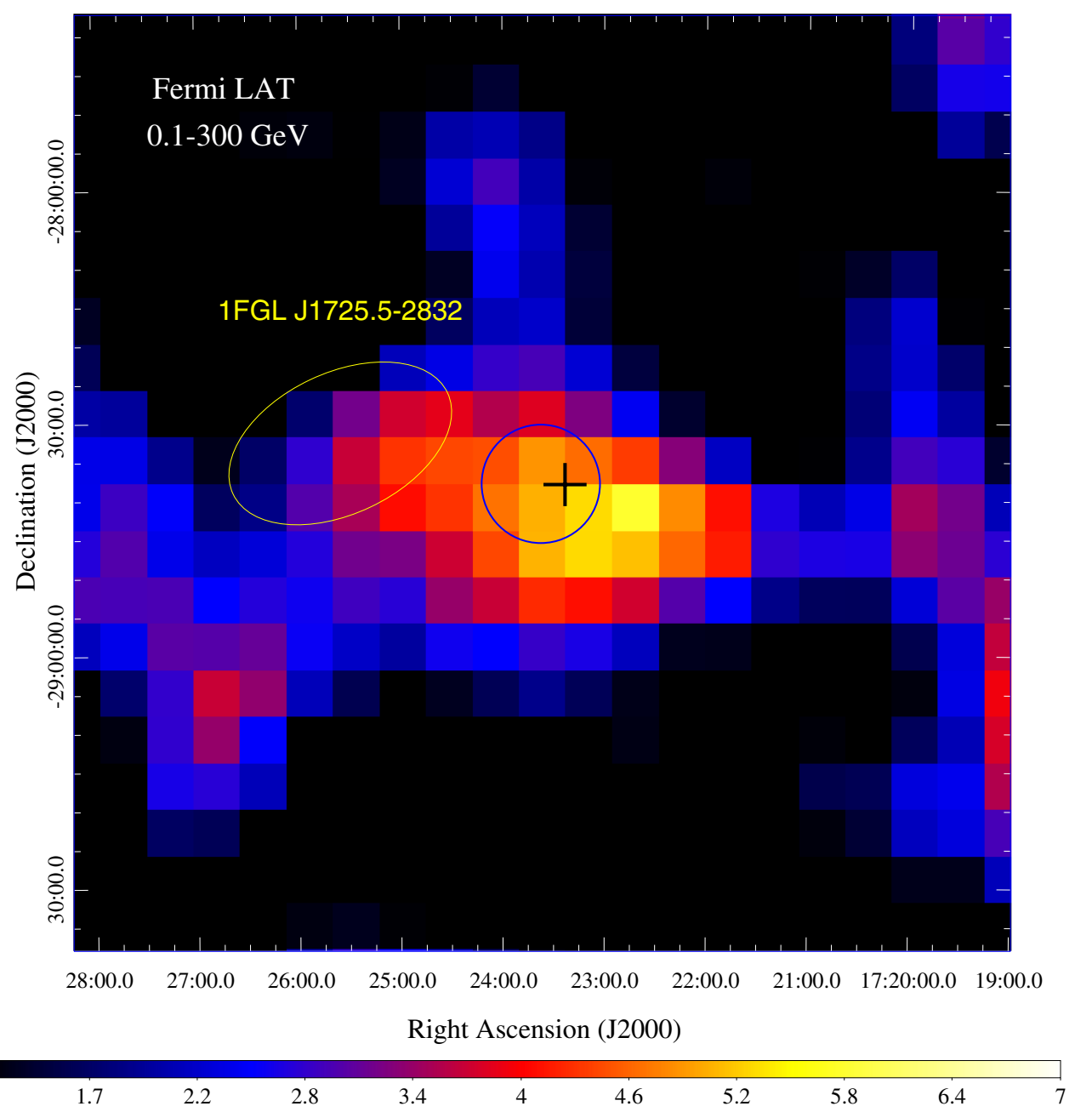

Figure 3. Background-subtracted $0.1-300 \mathrm{GeV} \gamma$-ray count map, smoothed with a Gaussian width of 0.3 , of the $2^{\circ} \times 2^{\circ}$ region centered on PSR J1723-2837, the radio timing position of which is indicated by the black cross. The blue circle indicates the error circle of the best-fit position at the $68 \%$ confidence level. The error eclipse of 1FGL J1725.5-2832, which is not regarded as a background source (see Section 2.3), is shown.

(A color version of this figure is available in the online journal.)

larger than $500 \mathrm{MeV}$ using gtfindsrc, resulting in a position at R.A.(J2000) $=260.906$, decl.(J2000) $=-28.631$, with statistical uncertainty 0.13 at the $68 \%$ confidence level, which is consistent with the position of PSR J1723-2837. The background-subtracted count map of the $2^{\circ} \times 2^{\circ}$ region centered on the radio position, constructed using the "clean" class events, is shown in Figure 3.

A nearby source in the first Fermi catalog (Abdo et al. 2010), 1FGL J1725.5-2832, is only $\sim 1^{\circ}$ away from PSR J1723-2837 (see Figure 3). We studied their relation by adding 1FGL J1725.5-2832 to the background source model which had been used in the aforementioned analysis. It turned out that essentially the same group of photons contributes to the TS values of both sources. Therefore, we treat 1FGL J1725.5-2832 as being the same $\gamma$-ray source associated with PSR J1723-2837.

Using the radio ephemeris reported in Crawford et al. (2013), we searched for possible $\gamma$-ray variability related to the orbital modulation. We divided the photons into two phase intervals, one centered at the SUPC (0.5-1.0), the other centered at the INFC (0.0-0.5), and performed binned likelihood analysis for each group of photons, using "source" events and the same analysis cuts and source model as described in the full-orbit analysis. No difference in the spectral parameters were found between SUPC and INFC. We also searched for any periodic signals using the $H$-test (de Jager \& Büsching 2010). Neither $\gamma$-ray periodicity related to the orbital period nor to the rotation period was found. However, the bright galactic diffuse emission, uncertainties in the ephemeris used over the Fermi data span, and small photon statistics from the source, make our periodicity searches less sensitive.

\section{DISCUSSION}

With a simple one-dimensional model (Takata et al. 2012), we constrain the pulsar wind parameters of PSR J1723-2837. Since the radio eclipse at several GHz lasts only $15 \%$ of its orbit (Crawford et al. 2013), we may expect that the shock wraps the companion star and its distance from the pulsar is on the order of the semimajor axis, which is $a \sim 3.7 \times 10^{10} / \sin i \mathrm{~cm}$, where $i$ is the orbital inclination angle. Based on the measurement of the Doppler velocity of the companion, Crawford et al. (2013) suggested the inclination angle of $i \sim 30^{\circ}-41^{\circ}$ for PSR J1723-2837. The radio eclipse also suggests the angle of flow from the companion star measured from the pulsar is $\theta_{a} \sim 2 \pi \times 0.15=0.94$. If the pulsar wind is emitted spherically, then the fraction of the pulsar wind stopped by the outflow is $\sim 5 \%$. The magnetic fields upstream $\left(B_{1}\right)$ and behind $\left(B_{2}\right)$ the shock are estimated as $B_{1} \sim\left(L_{\mathrm{sd}} \sigma / a^{2} c\right)^{1 / 2}$ 
and $B_{2}=3 B_{1}$, where $\sigma$ is the ratio of the magnetic energy to kinetic energy of the cold relativistic pulsar wind and we assume it to be smaller than unity (i.e., kinetically dominated flow). At the shock, the pulsar wind particles are accelerated beyond the Lorentz factor $\left(\Gamma_{1}\right)$ of the cold relativistic pulsar wind in the upstream region. For the maximum Lorentz factor of the accelerated particles, it is determined by balancing the accelerating timescale, $\tau_{\text {acc }} \sim \Gamma m_{e} c /\left(e B_{2}\right)$, and the synchrotron cooling timescale, $\tau_{s} \sim 9 m_{e}^{3} c^{5} /\left(4 e^{4} B^{2} \Gamma\right)$.

The observed X-ray photon index $\Gamma_{X} \sim 1.2$ may imply the power-law index of the particle distribution at the shock is $p \sim 1.4$. For the usual power-law index $p \sim 2-2.5$ of the shock acceleration, this hard photon index in the spectrum will be reproduced if the typical synchrotron energy $\left(E_{1}\right)$ of the minimum Lorentz factor $\Gamma_{1}$ is larger than $10 \mathrm{keV}$. The photons' index changes from $\alpha=(p+1) / 2$ above $E_{1}$ to $\alpha=3 / 2$ below $E_{1}$. In such a case, the X-ray emission properties break the degeneracy of $\sigma$ and $\Gamma_{1}$ in the emission model. For example, we can fit the observed properties $\alpha \sim 1.2$ and $f_{x} \sim 1.3 \times 10^{-12} \mathrm{erg} \mathrm{cm}^{-2} \mathrm{~s}^{-1}$ with $\left(\sigma, \Gamma_{1}\right) \sim\left(0.1,5 \times 10^{4}\right)$. The predicted Lorentz factor $\Gamma_{1} \sim 5 \times 10^{4}$ is similar to that of the original black widow pulsar PSR B1957+20 (Wu et al. 2012). As there is no evidence of a spectral break found in $0.3-10 \mathrm{keV}$, observations with state-of-the-art hard $\mathrm{X}$-ray telescopes, $N u S T A R$, and the upcoming Astro- $H$ will be important for constraining $E_{1}$.

Because PSR J1723-2837 follows an almost circular orbit, the shock distance from the pulsar does not vary across the orbit, suggesting that the spectral properties of the intrinsic shock emissions do not modulate with the orbital phase. The variation of the observed flux will be caused by either a Doppler boosting effect with a mildly relativistic flow of the shocked flow or by physical eclipse of the emission region. However, these two effects will not produce a significant variation in the spectrum. The observed amplitude (see Figure 1) implies that the Doppler factor is $\sim 1-2$, which does not cause a significant change in the spectral shape. Furthermore, the synchrotron cooling timescale of the particles that emit photons of energies $<10 \mathrm{keV}$ is $\tau_{s} \sim 33\left(B_{2} / 10 \mathrm{G}\right)^{-3 / 2}\left(E_{s} / 10 \mathrm{keV}\right)^{-1 / 2} \mathrm{~s}$, which is longer than the crossing timescale of the emission region, $\tau_{c} \sim 1 \mathrm{~s}$. The distribution of the particles and therefore the spectral shape of X-ray emission in $<10 \mathrm{keV}$ does not evolve in the emission region. As a result, even if the partial region of the emission region is covered by the star, the observed spectral shape is the same as that for whole emission regions.

For black widow/redback pulsars, the magnetospheric emissions and pulsar wind emissions produce the $\mathrm{GeV} \gamma$-rays. For the original black widow pulsar PSR B1957+20, Wu et al. (2012) suggested that both the magnetospheric and the pulsar wind emissions contribute to the $\mathrm{GeV}$ emissions seen by Fermi. With the results described in Section 2.3, it is unclear which process operates for the observed $\gamma$-rays for PSR J1723-2837, because neither the pulsar's spin period nor orbital modulation was found in the current Fermi data, and whether or not there is a cutoff in the spectrum is inconclusive. A single power-law feature in the observed spectrum in the $0.1-300 \mathrm{GeV}$ band would suggest that the $\gamma$-rays are emitted by the pulsar wind particles accelerated at the shock. In this case, the synchrotron radiation process explains the observed emissions around $100 \mathrm{MeV}$ and the inverse Compton scattering with the stellar photons contributes to the emissions above $\sim 10 \mathrm{GeV}$.

The shocked particles that emit the synchrotron photons will produce $\mathrm{TeV}$ photons through the inverse Compton off the stellar photons, for which the effective temperature is $T_{\text {eff }} \sim 4800-6000 \mathrm{~K}$. Based on the calculations with the isotropic photon fields, we can estimate the flux above $>100 \mathrm{GeV}$ as $F_{>100 \mathrm{GeV}} \sim 5 \times 10^{-13} \mathrm{erg} \mathrm{cm}^{-2} \mathrm{~s}^{-1}$, which could possibly be measured by the planned Cherenkov Telescope Array (CTA). The measurements by the CTA of the redback systems will provide us additional information such as the Lorentz factor of the relativistic pulsar wind and the maximum Lorentz factor of the shocked particles, etc.

C.Y.H. is supported by the National Research Foundation of Korea through grant 2011-0023383. P.H.T. is supported by the National Science Council of the Republic of China (Taiwan) through grant NSC101-2112-M-007-022-MY3. J.T. and K.S.C. are supported by a GRF grant of the HK Government under HKU7009 11P. A.K.H.K. is supported by the National Science Council of the Republic of China (Taiwan) through grants NSC100-2628-M-007-002-MY3 and NSC100-2923-M007-001-MY3. L.C.C.L. is supported by the National Science Council through grant NSC 101-2112-M-039-001-MY3.

\section{REFERENCES}

Abdo, A. A., Ackermann, M., Ajello, M., et al. 2010, ApJS, 188, 405 Archibald, A. M., Kaspi, V. M., Bogdanov, S., et al. 2010, ApJ, 722, 88 Archibald, A. M., Stairs, I. H., Ransom, S. M., et al. 2009, Sci, 324, 1411 Burderi, L., Possenti, A., D’Antona, F., et al. 2001, ApJL, 560, L71 Chen, H.-L., Chen, X. F., Tauris, T. M., \& Han, Z. W. 2013, ApJ, 775, 27 Crawford, F., Lyne, A. G., Stairs, I. H., et al. 2013, ApJ, 776, 20 de Jager, O. C., \& Büsching, I. 2010, A\&A, 517, L9

Mattox, J. R., Bertsch, D. L., Chiang, J., et al. 1996, ApJ, 461, 396

Nolan, P. L., Abdo, A. A., Ackermann, M., et al. 2012, ApJS, 199, 31 Papitto, A., Ferrigno, C., Bozzo, E., et al. 2013, Natur, 501, 517

Patruno, A., Archibald, A. M., Hessels, J. W. T., et al. 2013, ApJL, 781, L3 Predehl, P., \& Schmitt, J. H. M. M. 1995, A\&A, 293, 889

Roberts, M. S. E. 2013, in IAU Symp. 291, Neutron Stars and Pulsars: Challenges and Opportunities after 80 years, ed. J. van Leeuwen (Cambridge: Cambridge Univ. Press), 127

Shvartsman, V. F. 1970, R\&QE, 13, 1428

Takata, J., Li, K. L., Leung, G. C. K., et al. 2013, ApJ, submitted (arXiv:1312.0605)

Takata, J., Okazaki, A. T., Nagataki, S., et al. 2012, ApJ, 750, 70

Thorstensen, J. R., \& Armstrong, E. 2005, AJ, 130, 759

Wu, E. M. H., Takata, J., Cheng, K. S., et al. 2012, ApJ, 761, 181 